\title{
In the age of epigenetics, how do we define a gene?
}

\author{
Alexandra Fletcher \\ News Reporter (HSI 2014-2015)
}

Before Darwin, there was Lamarck. Lamarck believed in the inheritance of acquired characteristics; a theory that explored non-random phenotypic changes, but fell out of fashion with Darwin's Origin of Species ${ }^{1}$. The story that epitomizes Lamarckian evolution is that of the giraffe neck. In this instance, the giraffe was originally a short-necked creature that strengthened and lengthened its neck through consistent use, passing a longer, stronger neck down to its offspring. In contrast, Darwin believed in random mutations that may or may not increase an animal's likelihood of survival and reproduction. These mutations were more likely to be passed on if the animal survived and reproduced, but did not develop because of their inherent worth. It was cases of deviation from expected Mendelian inheritance patterns that hinted to scientists that epigenetic mechanisms must exist ${ }^{2}$.

This immutability of genetics, the idea of an inheritable set of laws embedded within our DNA, is gaining increasing acceptance ${ }^{3}$. Expressions such as "it's in your genes" have become commonplace in daily conversation. Despite this, epigenetics - the study of modifiable traits that are the result of environmental modifications -transforms this point of view and thereby gives a new outlook on the nature versus nurture debate. According to Dr. Guillaume Bourque, the Bioinformatics Director at McGill University and Génome Québec Innovation Centre, one way in which epigenetics can be comprehended that does not contradict public understanding of genetics is that every cell in the body contains the same genetic message, but not all cells have the same size, shape, or function. Cellular differentiation is therefore not due to different genes, but the microenvironment surrounding each cell, which results in some parts of the genome being activated while other parts are silenced. Epigenetics functions through a variety of chemical mechanisms, such as methylation and the addition of histone markers. To add a layer of complexity, these markers can be stable enough to be 'inherited' from one cell division to the next, but these changes are not permanently encoded into the genome. In the case of methylation, a methyl group $(\mathrm{CH} 3)$ is covalently bound to the nucleotide cytosine ${ }^{4}$. This methylation is conserved during DNA replication because of the action of the enzyme DNA methyltransferase, which is capable of recognizing a hemi-methylated DNA strand formed from the methylated parent strand and methylating an un-methylated daughter strand $^{4}$. In the case of histone modification, several chemical processes (including methylation) act on the histone protein responsible for binding DNA in its heterochromatic state, although the mechanism for maintaining modification during DNA replication is less clearly understood ${ }^{4}$. To further complicate the genetics/epigenetics paradigm, some of these chemical modifications can be passed on to offspring, though these effects are not as strong as those seen in genetic inheritance. Originally, epigenetic marks were thought of as being 'erased' either prior to or during meiosis, thereby limiting epigenetic changes to the individual who experienced them ${ }^{2}$. However, the evidence is now showing that 'soft inheritance' of epigenetic modifications is possible; in rat models, the treatment of gestational females with industrial chemicals leads to male infertility in subsequent generations ${ }^{5}$. And yet these two processes are distinct, as one is much more permanent than the other. One final subtlety within the description of epigenetics is that these modifications to the genome might also regulate higher-level interactions between distant DNA regions within or between chromosomes, rather than simply regulating individual genes ${ }^{2}$.

Another aspect of epigenetics that differs from genetics is that these changes occur over the course of someone's lifetime, and are therefore dependent upon the type of exposures an individual has had. The external factors capable of affecting which configuration of genes are turned on or off are still an active area of research. The field of epigenetics is still in its infancy, which means researchers such as Bourque still have many fundamental questions to answer. In his research, Dr. Bourque is working toward identifying what constitutes 


\section{HeAlTh SCIENCE INQUirY}

a 'normal' or 'baseline' state of a given cell. Only upon understanding this is it possible to begin asking what factors have the capacity to impact these baseline states. Identifying a baseline state is more complex than it may appear, given that the moment a cell begins to interact with its environment, it can be considered 'modified' by its surroundings. One way around this is to isolate a specific factor that can be studied across many individuals, and provide empirical evidence to substantiate the assumptions being made about the cell. The types of cohort studies to conduct this kind of research include natural experiments, longitudinal birth cohorts, longitudinal twin studies, prenatal cohorts, and in-vitro fertilization conception cohorts $^{6}$. These cohorts all aim to examine the effect of the environment and the genome at different points in time and space to see how epigenetic markers vary within and between individuals across time. The information derived from these types of studies can provide complementary evidence of the role of epigenetics (See Figure 1 for a graphical representation of study types). Much of the research in this field is also generated from studies using animal models or individual cell lines. Within this research, while it is much easier to precisely regulate the environment, the data generated are limited in their real-life applicability.

One of the features drug developers can take advantage of with respect to epigenetics is that it is not as definitive as genetics, which opens up the possibility of temporary and reversible modifications of the genome. Currently, there are drugs available that can modify one's epigenetic profile. This will become an important way to counteract disease states that alter the baseline profile of a cell, including some types of cancers. The first documented example of this was in colorectal cancer, where hypomethylated strands of DNA led to the over expression of oncogenes ${ }^{4}$. Currently, the research into therapeutic benefits has focused on globally inhibiting the mechanism of epigenetics by using methylation or histone acetylation inhibitors, which has the downfall of unintended effects of non-target genes. ${ }^{4}$ The alternative would be to target treatments toward the biochemical pathways that are selectively activated or silenced by epigenetic modifications ${ }^{3}$. The fact that it has
Epigenomic variation

$0 y \quad 40 y \quad 100 y$
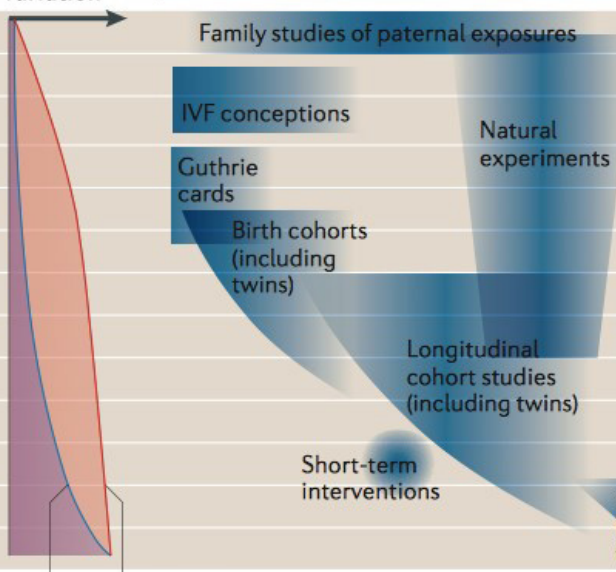

Guthrie cards Birth cohorts (including twins)

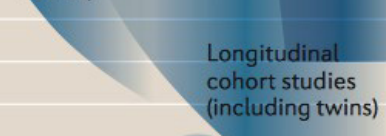
interventions Natura: experiments

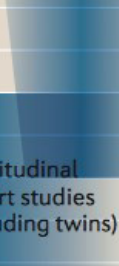

ongevity stochastic changes

Cumulative environmental changes

Figure 1. Description of study designs used in epigenetics and the information they provide. Taken from Mill, J. \& Heijmans, B.T. From promises to practical strategies in epigenetic epidemiology. Nat. Rev. Genet. 14, 585-594

been recognized that there are some diseases caused by epigenetic rather than genetic mutations suggests that potential therapies provide an exciting opportunity to have an significant impact on the course of disease.

\section{References}

1. Bird A. Genome Biology: Not Drowning but Waving. Cell 2013;154(5):95152.

2. Richards EJ. Inherited epigenetic variation - revisiting soft inheritance. Nat Rev Gen. 2006;7:395-401.

3. Koonin EV, Wolf YI. Is evolution Darwinian or/and Lamarckian? Biol Direct. 2009;4:42.

4. Feinberg A. Epigenetics at the Epicenter of Modern Medicine. J Am Med Assoc. 2008; 299(11):1345-50.

5. Anway MD, Cupp AS, Uzumcu M, Skinner MK. Epigenetic transgenerational actions of endocrine disruptors and male fertility. Science 2005;308:146669.

6. Mill J, Heijmans BT. From promises to practical strategies in epigenetic epidemiology. Nat Rev Genet. 2013;14:585-94. 


\section{Health Science InQuiry}

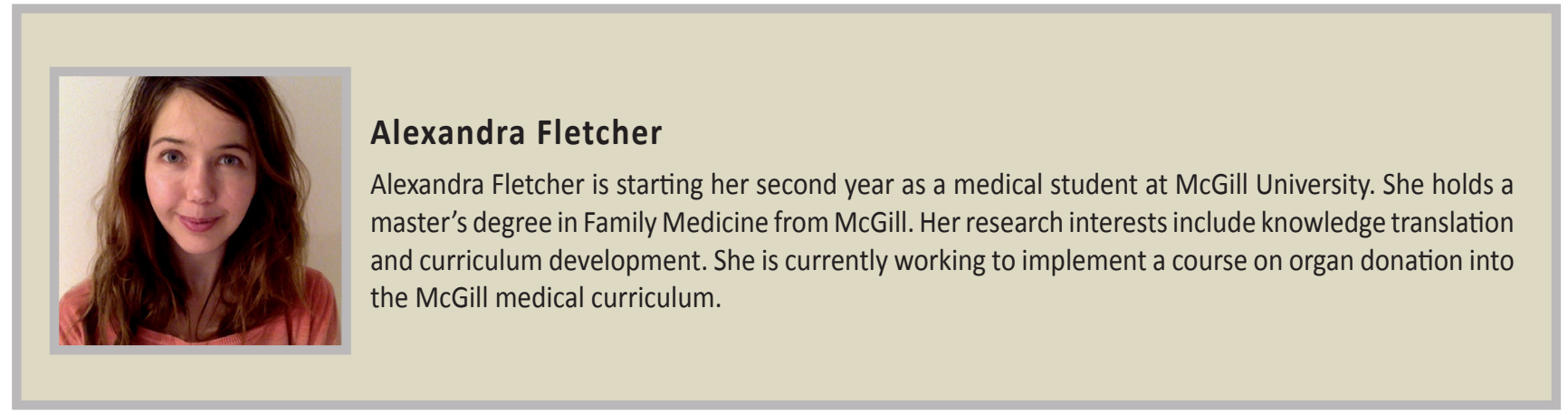

